\title{
Prospective, monocentric, repeated-measures study of ADHEAR in adults with middle ear disease
}

\author{
Rami Abu Dakah ${ }^{1}$, Dörte Fischer ${ }^{1}$, and Tino Just ${ }^{1}$ \\ ${ }^{1}$ KMG Klinikum Güstrow GmbH
}

January 17,2022

\begin{abstract}
Objective: The aim of this study was to evaluate the audiological performance of the ADHEAR system and to compare it with a softband BAHA system in adults with middle ear disease. Study Design: Prospective, single-subject, repeated-measures study Setting: Monocentric study Participants: In 23 patients with mild to moderate isolated conductive or combined hearing loss (CHL) the ADHEAR system was tested, where 12 of them were outside of the ADHEAR indication. In ten patients, testing was performed with the ADHEAR system and a softband BAHA system. A control group consisted of 10 patients with bilateral artificial occluded ear canal. Main outcome measures: Air and bone conduction thresholds and free-field monosyllable speech intelligibility thresholds in quiet and in noise were measured and evaluated in an unaided situation and aided situation. Furthermore, an ADHEAR questionnaire was assessed. Results: In patients with a middle ear disease compared to the unaided situation, the aided situations with the ADHEAR system and the softband BAHA system led to a significant improvement of speech intelligibility in quiet and in noise. In terms of the ADHEAR questionnaire, using the ADHEAR system patients reported about an improvement in sound localization, sound quality and speech intelligibility. Conclusions: The results show that patients with middle ear disease can benefit from the ADHEAR system, even if their hearing loss is outside of its indication. Outside the indication criteria of the ADHEAR system, it can be an option.
\end{abstract}

\section{Abstract \\ Objective:}

The aim of this study was to evaluate the audiological performance of the ADHEAR system and to compare it with a softband BAHA system in adults with middle ear disease.

\section{Study Design:}

Prospective, single-subject, repeated-measures study

\section{Setting:}

Monocentric study

\section{Participants:}

In 23 patients with mild to moderate isolated conductive or combined hearing loss (CHL) the ADHEAR system was tested, where 12 of them were outside of the ADHEAR indication. In ten patients, testing was performed with the ADHEAR system and a softband BAHA system.

A control group consisted of 10 patients with bilateral artificial occluded ear canal.

\section{Main outcome measures:}


Air and bone conduction thresholds and free-field monosyllable speech intelligibility thresholds in quiet and in noise were measured and evaluated in an unaided situation and aided situation. Furthermore, an ADHEAR questionnaire was assessed.

\section{Results:}

In patients with a middle ear disease compared to the unaided situation, the aided situations with the ADHEAR system and the softband BAHA system led to a significant improvement of speech intelligibility in quiet and in noise. In terms of the ADHEAR questionnaire, using the ADHEAR system patients reported about an improvement in sound localization, sound quality and speech intelligibility.

\section{Conclusions:}

The results show that patients with middle ear disease can benefit from the ADHEAR system, even if their hearing loss is outside of its indication. Outside the indication criteria of the ADHEAR system, it can be an option.

Key words: Adhear, bone conduction device, adhesive adapter, Baha

Five succinct key points.

The ADHEAR leads to similar hearing outcomes as a softband BAHA system in patients with combined hearing loss.

Patients with middle ear disease can benefit from the ADHEAR system, even if their hearing loss is outside of its indication.

The pressure less wearing comfort of this adhesive hearing device seems to be a permanent or temporary treatment option in patients who are unable to receive or deny middle ear surgery.

The ADHEAR system isn't always a permanent solution. For patients with middle ear disease, it is an option for the time period between the diagnosis and surgery or a hearing aid supply.

The softband BAHA system remains a solution for patients were adhesive wearing time is not satisfactory.

\section{Introduction}

For about four decades, a bone conduction (BC) hearing device may lead to hearing improvement in patients with single-sided deafness (SSD) and conductive or mixed hearing loss (CHL or MHL)(1). By vibration of the skull or structures of the middle ear, these devices transmit a signal directly into the cochlea.

$\mathrm{BC}$ hearing devices (BCHDs) can be divided into implantable and non-implantable devices. While implantable devices consist of an implant and an audio processor, with surgery necessary, non-implantable devices consist of an adapter and an audio processor, with surgery nor necessary $(2,3)$. For implantable BCHDs well known the percutaneous and transcutaneous bone-anchored hearing aids (BAHAs) like Ponto System and the BAHA $(\mathrm{B})$ Attract or Connect Systems(4), the transcutaneous osseointegrated steady-state system (Osia)(5) or the transcutaneous BC implant Bonebridge(r)(6), as well as the transcutaneous middle ear implant system Vibrant Soundbridge(r)(7). As non-implantable BCHDs, in 2001 a softband adapter for a BCHD audio processor was introduced, especially for children. Many studies showed that the softband BAHA is a successfully treatment for CHL(8-10). However, due to the static pressure on the head, some children and adults don't tolerate a softband as a long-term solution(11). As a new non-implantable BCHD was introduced, the ADHEAR(12,13). System, especially for small children(14-16). This system works without putting any pressure on the skin. The key concept of the ADHEAR system is a disposable adapter sticking to the hairless skin behind the ear, with an audio processor clicking onto this adapter. Previous studies showed that the audiometric results using the ADHEAR system are similar to those using a softband BAHA system(16), while the wearing time and acceptance in children and adults is preferred over a softband BAHA system $(12,16)$.

\section{Objective}


The aim for this study was to re-evaluate if adults with chronic middle ear disease of various etiologies may benefit from the ADHEAR system, regardless of its recommended indication criterion.

\section{Methods}

\subsection{Ethical considerations}

This study was conducted in strict adherence to the revised version of the Helsinki Declaration. The design of the study was approved by the Ethics Committee of the General Medical Council of Mecklenburg-West Pomerania (A2018-0035).

\subsection{Participants}

For participation, the inclusion criteria were German native speakers aged between 18 to 80 years with middle ear disease resulting in a CHL or MHL, unilateral or asymmetric hearing loss (HL). The results were compared with those of healthy subjects with a normal hearing ability. Patients with ear surgery of the contralateral ear, retrocochlear or central auditory disorders, skin diseases or other surgical conditions that may prevent the attachment of the adhesive adapter of the ADHEAR system were excluded.

Overall, 23 patients of the Department of Otorhinolaryngology, Head and Neck Surgery with middle ear disease (group 1) and 10 healthy volunteers as a control group participated in this prospective single-subject repeated-measure study (Table 1).

All subjects were informed about the aims of the study and provided their written consent.

\subsection{Procedure}

All patients and healthy subjects obtained a detailed medical report and an ear, nose and throat examination. The patients with middle ear disease were identified by otoscopy. In all healthy subjects, a chronic otitis media was excluded.

All participants obtained a pure tone audiometry and free-field speech audiometry under various conditions, i.e., in the unaided situation and aided situations using the ADHEAR system and the softband BAHA 5 Power system. Furthermore, to assess the hearing ability before and after using the ADHEAR system, all patients were asked to fill out the questionnaire.

\subsection{Outcome measures}

All audiological assessments were conducted in an audiometric sound attenuated room, using calibrated signals and equipment according to accepted ISO standard. For assignment of the participants a pure tone audiometry was performed measuring the air and bone conduction thresholds at $0.5,1,2,3,4,6$, and $8 \mathrm{kHz}$. The PTA4 was calculated for the bone conductive and air tone thresholds across the frequencies of $0.5,1,2$, and $4 \mathrm{kHz}$.

The speech intelligibility tests were conducted in the free field using the Freiburger monosyllables word test in quiet and in noise. The loudspeakers were positioned $1 \mathrm{~m}$ away from the participants' head. In quiet, i.e., under the $\mathrm{S}_{0}$ condition, the speech intelligibility was measured at $65 \mathrm{~dB}$ SPL. In noise, i.e., under the $\mathrm{S}_{0} \mathrm{~N}_{0}$ condition, the speech intelligibility was measured with a fixed noise level at $60 \mathrm{~dB}$ SPL and a speech level at $65 \mathrm{~dB}$ SPL, resulting in a signal-to-noise ratio (SNR) of $5 \mathrm{~dB}$. All speech intelligibility measurements were performed in the unaided situation and the aided situation with the ADHEAR system. In order to compare the performance of the ADHEAR system, 10 patients and the 10 healthy subjects were also fitted with a softband BAHA system using the BAHA 5 Power system.

In order to measure the speech intelligibility of the ipsilateral ear as isolated as possible, the contralateral ear was masked using an earplug (Oropax(r)) if applicable.

In the control group, the healthy subjects with normal or age-appropriate hearing ability, both ears were plugged with an earplug (Oropax(r)) reaching 40 +- $8.2 \mathrm{~dB}$ CHL (ranging 22.5-50 dB). 
A questionnaire focused on several aspects encountered in applying the ADHEAR system, namely handling of the system, hearing sensation and problems.

\subsection{Data analyses}

Statistical tests (Kolmogorov Smirnov test and Shapiro-Wilks test) indicated non-normal distribution for all parameters. Nonparametric tests were performed for group comparisons whenever appropriate. Pearson statistics were used for correlational analyses. The alpha level was set to 0.05. SPSS 27.0 (SPSS Inc. Chicago, IL, USA) was used for statistical analyses. In this paper, we will only discuss significant results.

\section{Results}

\subsection{Group 1 (Middle Ear Disease)}

For group 1, the 23 patients with middle ear disease, the measured PTA4 values for the air and bone conduction HL are presented in Fig 1. The dotted and the dashed lines point the indication criteria for the ADHEAR system (BC PTA4 [?] $25 \mathrm{~dB}$ HL) and for the BAHA 5 Power system (BC PTA4 [?] $55 \mathrm{~dB} \mathrm{HL}$ ), respectively. Fig 1 shows that 23 patients were aided with the ADHEAR system, while the HL of only 11 patients is within the ADHEAR criterion. Ten patients were aided with both the ADHEAR and the BAHA 5 Power system, while the HL of 6 patients is within the ADHEAR criterion.

\section{Pure Tone Audiometry}

The mean BC threshold was $28+-17 \mathrm{~dB}$ HL and the air bone gap on the affected side was $29+-14 \mathrm{~dB}$ HL. On the contralateral side, a mean PTA4 of $22+-16.5 \mathrm{~dB}$ HL and an air bone gap of $9.5+-11 \mathrm{~dB}$ HL was found.

\section{Speech Audiometry in Quiet and in Noise}

Table 2 depicts the speech intelligibility results of the free-field Freiburg monosyllables word test in quiet at $65 \mathrm{~dB}$ SPL and in noise at $5 \mathrm{~dB}$ SNR, for the unaided situation and the aided situations using the ADHEAR system and the BAHA 5 Power system. The mean speech intelligibility score in the unaided situation in quiet was $33.0+-36.1 \%$, which is significantly lower than using a softband BAHA system $65+-32.5 \%$ or the ADHEAR system $51.5+-32.4 \%$ (all p's $<0.05$ ). In terms of the speech intelligibility test in noise under $\mathrm{S}_{0} \mathrm{~N}_{0}$ condition, the mean thresholds of the unaided situation at $65 \mathrm{~dB}$ SPL were $17.4+-23.4 \%$. Using a softband BAHA system $(32.0+-29.2 \%)$, the mean thresholds in noise were significantly improved compared to the unaided situation (all p's $<0.05)$. Using the ADHEAR system, the mean threshold $(30.4+-28.0 \%)$ was highly significantly improved compared to the unaided situation $(\mathrm{p}<0.001)$.

Figs $2 \mathrm{~A}$ and $\mathrm{B}$ depict the speech intelligibility scores of the 23 ADHEAR patients (see Fig. 1) for the unaided situation and the aided situation in quiet at $65 \mathrm{~dB}$ SPL and in noise at $5 \mathrm{~dB}$ SNR, respectively. In quiet, significant differences were found in patients outside the ADHEAR indication criterion (BC PTA4 $>25 \mathrm{~dB}$ HL) $(\mathrm{p}<0.05$; see Fig. 2A). In noise, significant differences were found in both within and outside the indication criterion of the ADHEAR system (all p's $<0.05$ ). Figs 3A and B depict the speech intelligibility scores of the 10 ADHEAR \& BAHA 5 Power patients (see Fig. 1) for the unaided situation and the aided situations in quiet and in noise, respectively.

\subsection{Control Group (Healthy Subjects)}

In terms of the Freiburg monosyllables word test in quiet at $65 \mathrm{~dB}$ SPL, the mean speech intelligibility score in the unaided situation $(40.0 \%+-15.5 \%)$ was significantly improved to $75 \%+-11.3 \%$ using the BAHA 5 Power system $(\mathrm{p}<0,01)$ and to $73.0+-15.9 \%$ using the ADHEAR system $(\mathrm{p}<0.01)$.

At $5 \mathrm{~dB}$ SNR, the speech intelligibility scores in the unaided situation decreased from $40.0 \%+-15.5 \%$ in quiet to $19.0+-12.4 \%$. The mean speech intelligibility score in the unaided situation was significantly improved to $49.0 \%$ +- $9.7 \%$ using the BAHA 5 Power system $(\mathrm{p}<0,01)$, and to $41.0+-23.2 \%$ using the ADHEAR system $(\mathrm{p}<0.05)$. 


\subsection{Questionnaire}

In summary of the questionnaire, three patients of group 1 and two healthy subjects of the control group reported on problems with the adhesive adapter over time. Main factors were sweating and leisure activities. The lack of adhesion leads to an inadequate coupling and inadequate transmission. However, no adverse reactions against the adhesive adapter were reported. Most of the participants (70\%) reported on sound quality and speech understanding, similar to a softband BAHA.

\section{Discussion}

In our study the ADHEAR system was applied to an inhomogeneous group of patients with middle ear diseases of multiple etiologies suffering from a uni- or bilateral CHL or MHL. As a control group, the ADHEAR system was applied to healthy subjects with an artificial occluded outer ear canal. The patients and healthy subjects were aided with the ADHEAR system regardless of the indication criterion of the system.

In patients with a middle ear disease, 10 patients aided with a softband BAHA 5 Power system and the ADHEAR system led to a significant improvement of speech intelligibility in quiet and in noise, compared to the unaided situation. Similar results were found in a pilot study by Skarzynski et al.(17). Another study on 11 patients after middle ear surgery showed a significant improvement of speech intelligibility for monosyllables in quiet by $46 \%$ with the ADHEAR system compared to the unaided situation. The authors concluded that this adhesive BCHD may considerably improve treatment of patients with even short-term HL.

In patients with chronic inflammation of the outer ear canal and/or the middle ear, a conventional hearing aid is often refused by the doctor during conservative treatment. Even after middle ear surgery or surgery of the outer ear canal there is also a need for a temporary alternative for hearing improvement. In these cases, a softband BAHA system or an adhesive BCHD may be an alternative to improve hearing.

A softband BAHA system is sometimes refused by the patients due to social stigmatization and sweating, especially in summer. Therefore, we initialized the study starting in summer to prove whether the ADHEAR system is an alternative to a softband BAHA system or not.

According to the company information, the adhesive adapter is designed for single use and can remain on the skin for 3-7 days $(9,12,15,18)$. Considering the statements in the questionnaires, in four out of 33 participants of the study, the adhesive adapter detached more than 2 times per day due to sweating. The reported daily use and the comfort level was high, suggesting good patients' acceptance. Additionally, no allergic reactions or other skin alteration were observed in all participants. However, for a few patients the adhesive solution is not satisfactory such that the softband BAHA remains an alternative. Patients with long-time experience for the ADHEAR and the softband BAHA system reported a higher wearing comfort and longer wearing time with the ADHEAR system than the softband BAHA system. Similar results were reported by Neumann et al.(16) and Dahm et al.(18). More data are needed to conclude that an adhesive solution is the preferred option.

\section{Conclusions:}

Our study results allow the conclusion that the decision with modality can be recommended to improve hearing in patients with middle ear disease is not only audiologic but involves both audiological and clinical assessment. Hearing results with the adhesive ADHEAR system within and outside its indication criterion were compared to the softband bone anchored hearing aid (BAHA) system in patients with conductive or mixed hearing loss. Due to the pressure-less wearing comfort we see the ADHEAR system as a permanent or temporary treatment option in patients who are unable to receive or deny surgery. Outside the indication criterion of the ADHEAR system, the use of the ADHEAR system can be an option. Softband BAHA systems remain a solution for patients were the adhesive wearing time is not satisfactory.

Figures 
Fig. 1 .

Air conduction (AC) and bone conduction (BC) hearing levels (PTA4) for patients with middle ear disease (CHL or MHL - group 1). The dotted line points the indication criterion of the ADHEAR system at a BC of less than $25 \mathrm{~dB}$ HL and the dashed line points the indication criterion of the BAHA 5 Power system at a $\mathrm{BC}$ less than $55 \mathrm{~dB}$ HL.

Fig. 2A

Speech intelligibility scores (free-field Freiburg monosyllables word test results) in quiet at 65 dB SPL for the unaided situation and the aided situation with the ADHEAR system for patients in group 1 (middle ear disease) within and outside the indication criterion for ADHEAR (in: $\mathrm{n}=11$ and out: $\mathrm{n}=12$ ).

Fig. 2B

Speech intelligibility scores (free-field Freiburg monosyllables word test results) in noise at 5 dB SNR for the unaided situation and the aided situation with the ADHEAR system for patients in group 1 (middle ear disease) within and outside the indication criterion for ADHEAR (in: $\mathrm{n}=11$ and out: $\mathrm{n}=12$ ).

Fig. 3A

Speech intelligibility scores (free-field Freiburg monosyllables word test results) in quiet at 65 dB SPL for the unaided situation and the aided situations with the ADHEAR system and the softband BAHA 5 Power system for patients in group 1 (middle ear disease) within and outside the indication criterion for ADHEAR (in: $\mathrm{n}=6$ and out: $\mathrm{n}=4$ ).

Fig. 3B

Speech intelligibility scores (free-field Freiburg monosyllables word test results) in noise at 5 dB SNR for the unaided situation and the aided situations with the ADHEAR system and the softband BAHA 5 Power system for patients in group 1 (middle ear disease) within and outside the indication criterion for ADHEAR (in: $\mathrm{n}=6$ and out: $\mathrm{n}=4$ ).

Tables

Table 1. Demographics and baseline characteristics for all patients with middle ear disease and for the control group (subjects with bilateral plugged ears).

Table 2. Mean speech intelligibility differences between the unaided situation and the aided situations, using the ADHEAR system and a softband BAHA 5 Power system.

1. Tjellstrom A, Hakansson B, Granstrom G. Bone-anchored hearing aids: current status in adults and children. Otolaryngol Clin North Am. 2001;34(2):337-64.

2. Cedars E, Chan D, Lao A, Hardies L, Meyer A, Rosbe K. Conversion of traditional osseointegrated boneanchored hearing aids to the Baha $((\mathrm{R}))$ attract in four pediatric patients. Int J Pediatr Otorhinolaryngol. 2016;91:37-42.

3. Gawecki W, Stieler OM, Balcerowiak A, Komar D, Gibasiewicz R, Karlik M, et al. Surgical, functional and audiological evaluation of new Baha((R)) Attract system implantations. Eur Arch Otorhinolaryngol. 2016;273(10):3123-30.

4. Oberlies NR, Castano JE, Freiser ME, McCoy JL, Shaffer AD, Jabbour N. Outcomes of BAHA connect vs BAHA attract in pediatric patients. Int J Pediatr Otorhinolaryngol. 2020;135:110125.

5. Mylanus EAM, Hua H, Wigren S, Arndt S, Skarzynski PH, Telian SA, et al. Multicenter Clinical Investigation of a New Active Osseointegrated Steady-State Implant System. Otol Neurotol. 2020;41(9):1249-57.

6. Sprinzl G, Lenarz T, Ernst A, Hagen R, Wolf-Magele A, Mojallal H, et al. First European multicenter results with a new transcutaneous bone conduction hearing implant system: short-term safety and efficacy. 
Otol Neurotol. 2013;34(6):1076-83.

7. Labassi S, Beliaeff M, Pean V, Van de Heyning P. The Vibrant Soundbridge((R)) middle ear implant: A historical overview. Cochlear Implants Int. 2017;18(6):314-23.

8. Hol MK, Cremers CW, Coppens-Schellekens W, Snik AF. The BAHA Softband. A new treatment for young children with bilateral congenital aural atresia. Int J Pediatr Otorhinolaryngol. 2005;69(7):973-80.

9. Osborne MS, Child-Hymas A, McDermott AL. Longitudinal study of use of the pressure free, adhesive bone conducting hearing system in children at a tertiary centre. Int J Pediatr Otorhinolaryngol. 2020;138:110307.

10. Verhagen CV, Hol MK, Coppens-Schellekens W, Snik AF, Cremers CW. The Baha Softband. A new treatment for young children with bilateral congenital aural atresia. Int J Pediatr Otorhinolaryngol. 2008;72(10):1455-9.

11. Reinfeldt S, Hakansson B, Taghavi H, Eeg-Olofsson M. New developments in bone-conduction hearing implants: a review. Med Devices (Auckl). 2015;8:79-93.

12. Dahm V, Baumgartner WD, Liepins R, Arnoldner C, Riss D. First Results With a New, Pressure-free, Adhesive Bone Conduction Hearing Aid. Otol Neurotol. 2018;39(6):748-54.

13. Dobrev I, Farahmandi TS, Huber AM, Roosli C. Experimental Evaluation of the Adhear, a Novel Transcutaneous Bone Conduction Hearing Aid. Laryngorhinootologie. 2021;100(10):811-7.

14. Favoreel A, Heuninck E, Mansbach AL. Audiological benefit and subjective satisfaction of children with the ADHEAR audio processor and adhesive adapter. Int J Pediatr Otorhinolaryngol. 2020;129:109729.

15. Hirth D, Weiss R, Stover T, Kramer S. Audiological benefit and subjective satisfaction with the ADHEAR hearing system in children with unilateral conductive hearing loss. Eur Arch Otorhinolaryngol. 2021;278(8):2781-8.

16. Neumann K, Thomas JP, Voelter C, Dazert S. A new adhesive bone conduction hearing system effectively treats conductive hearing loss in children. Int J Pediatr Otorhinolaryngol. 2019;122:117-25.

17. Skarzynski PH, Ratuszniak A, Osinska K, Koziel M, Krol B, Cywka KB, et al. A Comparative Study of a Novel Adhesive Bone Conduction Device and Conventional Treatment Options for Conductive Hearing Loss. Otol Neurotol. 2019;40(7):858-64.

18. Dahm V, Auinger AB, Liepins R, Baumgartner WD, Riss D, Arnoldner C. A Randomized Crossover Trial Comparing a Pressure-free, Adhesive to a Conventional Bone Conduction Hearing Device. Otol Neurotol. 2019;40(5):571-7. 

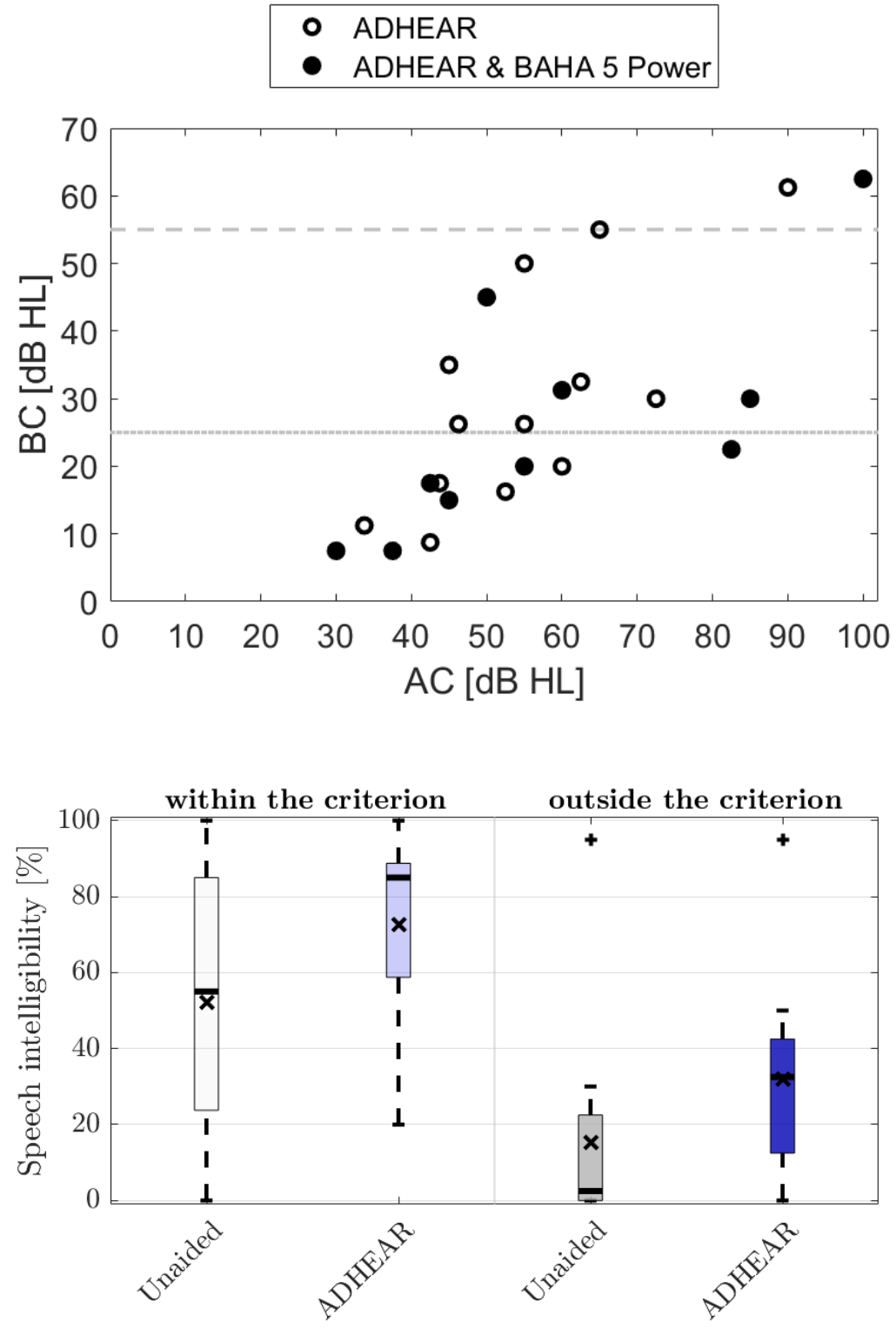

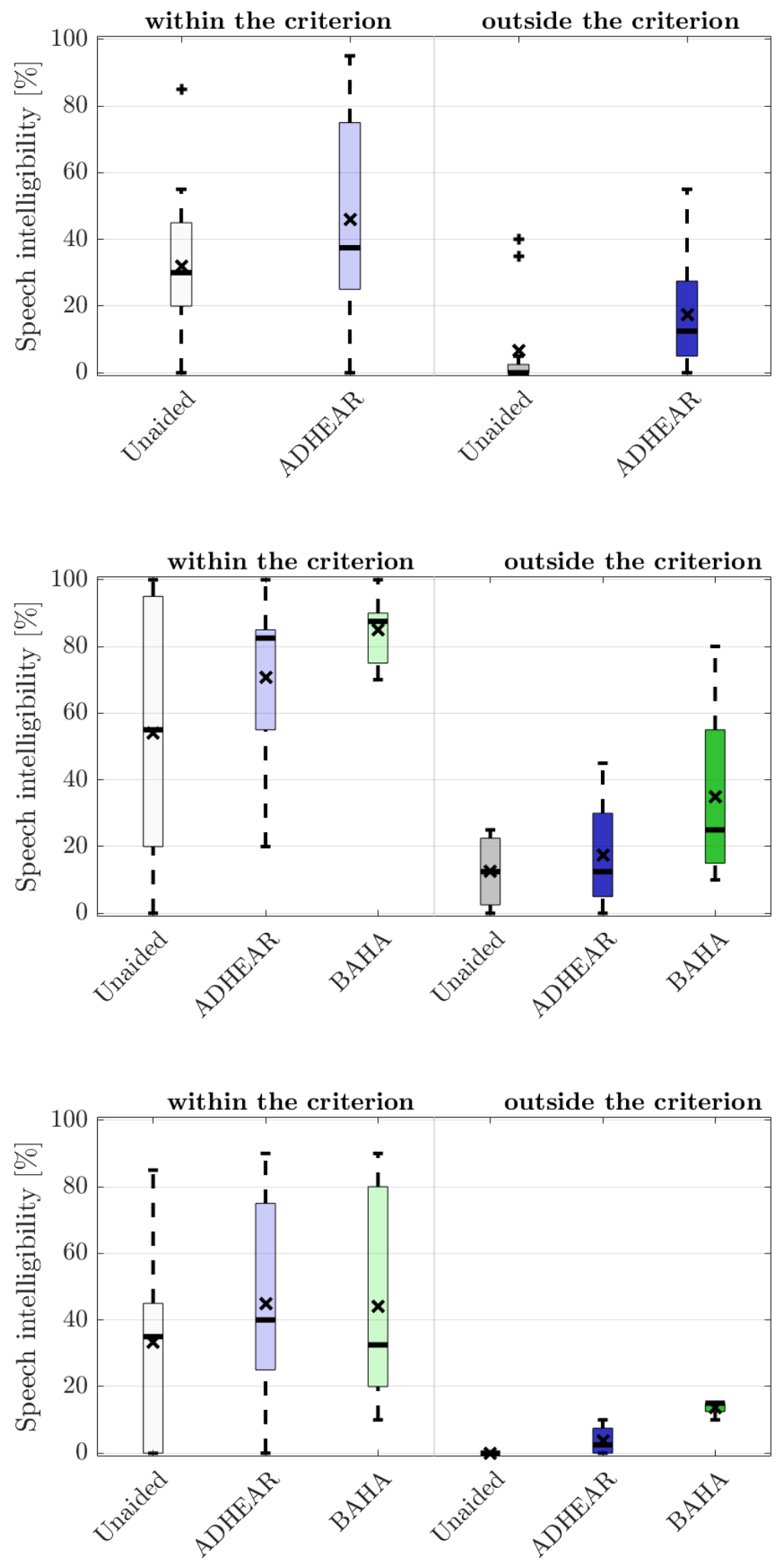

Hosted file 
Table 1.docx available at https://authorea.com/users/455830/articles/553074-prospectivemonocentric-repeated-measures-study-of-adhear-in-adults-with-middle-ear-disease

\section{Hosted file}

Table2.docx available at https://authorea.com/users/455830/articles/553074-prospectivemonocentric-repeated-measures-study-of-adhear-in-adults-with-middle-ear-disease 